\title{
Influence of surface based cohesive parameters on static performance of concrete composite T-shaped beams
}

\author{
Łukasz Jabłoński ${ }^{1, *}$, Anna Halicka ${ }^{1}$ \\ ${ }^{1}$ Lublin University of Technology, Faculty of Civil Engineering and Architecture, Nadbystrzycka 40, Lublin 20-618, Poland
}

\begin{abstract}
The study presented constitutes the next step of authors' investigations on the concrete composite T-shaped beams. The comparison of the failure modes and crack patterns of numerical models and beams tested was performed previously. In this paper, analyses concentrate on determination the proper values of cohesive surface parameters ensuring the high conformity of deformation and strains of the numerical model to the experimental results. In the particular series of beams the interfaces between concrete parts are varied as follows: reinforced joint with normal and tangential interactions only (BZ/S2/A), non-reinforced joint with adhesion (BZ/P), and reinforced joint with adhesion (BZ/P+S). Material properties used in numerical model are based on obtained in the laboratory ones. Furthermore, the 'traction-separation' law in the ABAQUS software defining the cohesion surface is used and analysis of the cohesive parameters response to interface roughness is carried out. The results show a strong dependence between the cohesion surface parameters and the response of the composite beam. A very good convergence of the numerical model with the experimental results in terms of 'force-displacement' relationship is achieved. Finally the parameters defined in the ABAQUS are determined, which have a key impact on the beams response.
\end{abstract}

\section{Introduction}

Concrete composite elements are commonly used in bridges, rib-and-slab floors and many precast buildings. Higher bearing capacity in comparison to bearing capacity of separate parts leads to increasingly more applications of such elements nowadays. Composite elements are characterized by existence of interface, most often the weakest zone with its own strength depending on the surface arrangement. Therefore the recognition of interface parameters and determining those of the key impact on static performance of such elements seems to be very important [1-6]. That is why the authors undertook laboratory tests of concrete composite T-shaped beams with variously arranged interface located between web and flange: adhesive reinforced interface, adhesive interface without reinforcement, and reinforced interface with limited or broken adhesion [1-3].

The initial numerical analyses of beams tested were executed using Finite Element Method software ABAQUS/Standard [1]. They were concentrated on the crack patterns. The stiffness degradation parameter (SDEG), which simulates the crack formation process in the numerical model was compared with cracks obtained experimentally. They were similar [1].

Defining the interface in concrete composite element as a cohesive surface seems to be suitable for the global static performance modelling. In this paper, analyses, carried out using also ABAQUS/Standard software, are focused on the determination the proper values of cohesive surface parameters, ensuring the high conformity of the numerical model to the experimental results. The deflection and the reinforcement strains are analysed for such purpose.

Numerical characteristics of materials used in models are based on material properties obtained in the laboratory tests. The numerical characteristics of interfaces vary in particular models reflecting different beams series tested. In all models, concrete parts are independent, but normal and tangential behaviours are regarded. The value of friction parameter $\mu$ responds to the roughness of the surface. Additional technique (Cohesive Zone Method), enabling parts separation in order to simulate cracking and delamination in the interface is used in series, in which adhesion is present. The 'traction-separation' $(t-s)$ law is used to describe cohesive behaviour. In ABAQUS, the bilinear $t-s$ law is defined as: an elastic range of interface, the critical point corresponding to the start of damage and complete material separation corresponding to the end of damage. Therefore the parameters of $t-s$ law are: traction damage initiation stresses $t_{n n}^{o}, t_{s s}^{o}, t_{t t}^{o}$, cohesive surface stiffness $K_{n n}^{o}, K_{s s}^{o}, K_{t t}^{o}$, separation displacement at failure $\delta_{n n}^{f}, \delta_{s s}^{f}$, $\delta_{t t}^{f}$. The process associated with proper selection of the $K^{o}$ and $t^{o}$ parameters is time consuming because they do not have their equivalents in real materials constants and are only virtual values used to calibrate a numerical model.

\footnotetext{
* Corresponding author: jablonski@pollub.pl
} 
The study presented concentrates on impact of parameters defined in the ABAQUS program on the response of beams tested. The purpose is to achieve the best correlation with the laboratory tests results. Also, analysis is performed to find parameters of the key impact on response and their value ranges which can be used in further investigations [7-12].

\section{Methodology}

\subsection{Laboratory tested members}

The T-shaped composite beams with the variously arranged interface, located between the web and flange were tested. Their dimensions were: $1800 \mathrm{~mm}$ in span length, $640 \times 50 \mathrm{~mm}$ flange cross-section, $80 \times 150 \mathrm{~mm}$ web cross-section (Fig. 1). The beams were designed to be damaged in the support zones. The procedure of beam production was divided into two stages. First, the concrete mix forming the web was placed in the mould. Next, after 14 days of concrete curing, treatment of "old" concrete surface (moisturizing and roughening with wire brushes) was made and then the mould was completed with a layer of "new" concrete. The longitudinal reinforcement consisted of two $\varnothing 14 \mathrm{~mm}$ bars (the longitudinal reinforcement ratio was $2.21 \%$ ), while the upper reinforcement consisted of two $\varnothing 8 \mathrm{~mm}$ bars. Stirrups made of Ø4 $\mathrm{mm}$ wires were used.

There were five series of beams with the interface arranged as follows:

- $\quad \mathrm{BZ} / \mathrm{P}+\mathrm{S}-$ reinforced interface $\left(\rho_{\mathrm{i}}=0.21 \%\right)$ with adhesion,

- $\quad \mathrm{BZ} / \mathrm{P}-$ non-reinforced interface with adhesion,

- $\quad \mathrm{BZ} / \mathrm{S} 1$ - reinforced interface $\left(\rho_{\mathrm{i}}=0.21 \%\right)$ with the surface of "old" concrete treated using chemical agent in order to limit the adhesion,

- $\quad \mathrm{BZ} / \mathrm{S} 2$ - reinforced interface (series BZ/S2/A $\rho_{\mathrm{i}}=0.21 \%$, series $\left.\quad \mathrm{BZ} / \mathrm{S} 2 / \mathrm{B} \quad \rho_{\mathrm{i}}=0.42 \%\right)$ with completely broken adhesion using utilization of PVE membrane.

The beams were tested as simply supported in fourpoint test. In order to ensure stability, the beams were inverted upside down and the load was applied using a rigid traverse. In each series three beams were tested. The load of one beam in each series was constantly increased with $0.5 \mathrm{kN} / \mathrm{min}$. For the other beams in the series, the load was stopped at intervals of $10 \mathrm{kN}$ to register the width and pattern of cracks. Deflections and strain of bars were measured automatically by the LVTD and strain gauges.

The steel and concrete parameters of the "old" and "new" layer are listed in Table 1.

\subsection{Numerical model}

In the Finite Element Method analysis, presented below, the beams of series BZ/S2/A (reinforced interface with broken adhesion), BZ/P (non-reinforced interface with adhesion) and $\mathrm{BZ} / \mathrm{P}+\mathrm{S}$ (reinforced interface with adhesion) are modelled.

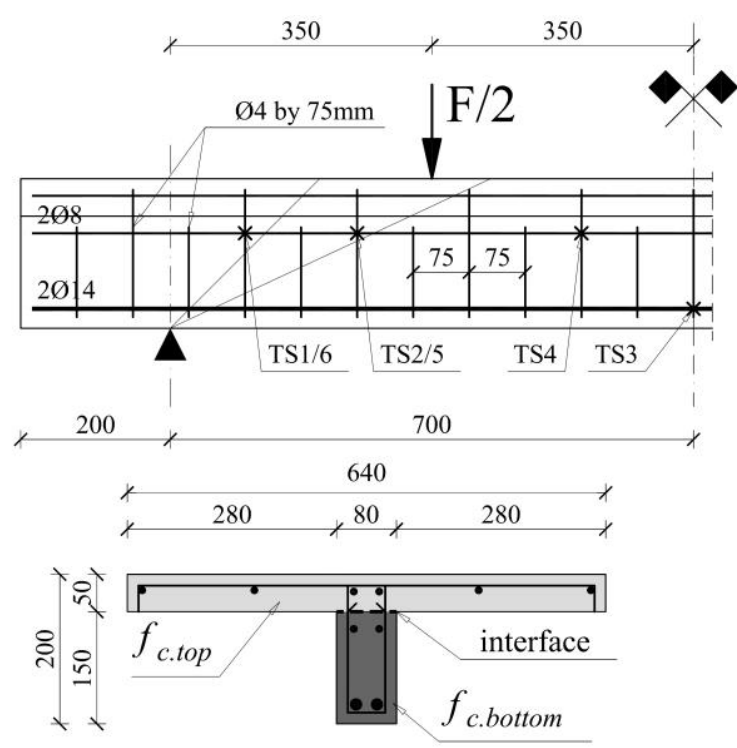

Fig. 1. Details of tested beams: a) longitudinal section, b) cross-sections; TS1,TS2 - strain gauges on the stirrups, TS3 - strain gauges on the main bar.

Table 1. Average values of material parameters.

\begin{tabular}{|c|c|c|c|}
\hline Specimen & $\begin{array}{c}\text { Compressive } \\
\text { strength } \boldsymbol{f}_{\text {cm }} \\
{[\mathbf{M P a}]}\end{array}$ & $\begin{array}{c}\text { Tensile } \\
\text { strength } \boldsymbol{f}_{\text {ctm }} \\
\text { [MPa] }\end{array}$ & $\begin{array}{c}\text { Modulus of } \\
\text { elasticity } \boldsymbol{E}_{\boldsymbol{c m}} \\
\text { [GPa] }\end{array}$ \\
\hline \multicolumn{4}{|c|}{ BZ/P+S series } \\
\hline 'Top' layer & 44.37 & 3.36 & 34.40 \\
\hline 'Bottom' layer & 47.01 & 3.57 & 35.00 \\
\hline \multicolumn{4}{|c|}{ BZ/P series } \\
\hline 'Top' layer & 41.54 & 3.18 & 33.73 \\
\hline 'Bottom' layer & 48.87 & 3.43 & 35.41 \\
\hline \multicolumn{4}{|c|}{ BZ/S1 series } \\
\hline 'Top' layer & 42.76 & 3.06 & 34.02 \\
\hline 'Bottom' layer & 45.55 & 3.04 & 34.67 \\
\hline \multicolumn{4}{|c|}{ BZ/S2/A and BZ/S2/B series } \\
\hline 'Top' layer & 54.72 & 3.49 & 36.63 \\
\hline 'Bottom' layer & 57.25 & 3.56 & 37.13 \\
\hline Stirrup yield stress $f_{\text {ywk }[\mathrm{MPa}]}$ & 340 \\
\hline $\begin{array}{l}\text { Longitudinal reinforcement yield stress } \\
\text { fyk [MPa] }\end{array}$ \\
\hline $\begin{array}{l}\text { Modulus of elasticity of reinforcement } \\
E_{s}[\mathrm{GPa}]\end{array}$ \\
\hline
\end{tabular}

The model of the concrete beam (Fig. 2) is built of the C3DR8 standard linear solid elements. The B31 beam elements are used to model the reinforcement. Numbers of elements and nodes are listed in the Table 2. The mesh size is approximately of $10 \mathrm{~mm}$. The symmetry of beam is regarded and therefore a quarter of beam was modelled.

The Concrete Damaged Plasticity inbuilt model is adopted, whereas for steel an "elastic-ideal plastic" model is used [7, 9-12]. The CDP model based on the material properties is listed in Table 1. 


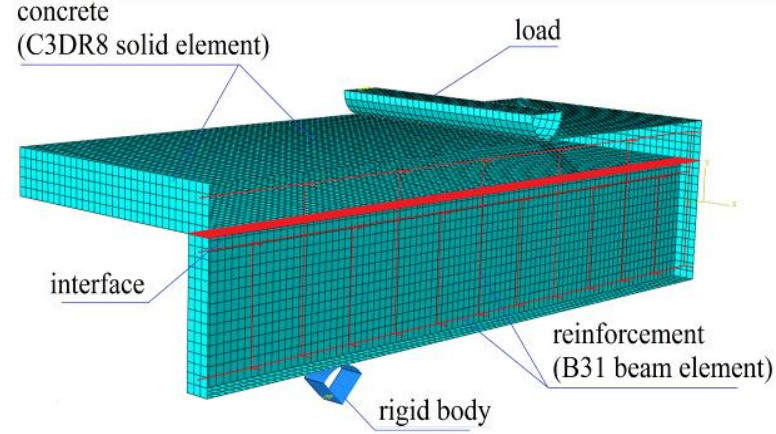

Fig. 2. Discrete 3D model and applied boundary conditions.

Table 2. Element type, number of elements and nodes.

\begin{tabular}{|c|c|c|c|}
\hline Component & FE Type & $\begin{array}{c}\text { No. } \\
\text { Elements }\end{array}$ & $\begin{array}{c}\text { No. } \\
\text { Nodes }\end{array}$ \\
\hline $\begin{array}{c}\text { Web and } \\
\text { flange }\end{array}$ & $\begin{array}{c}\text { C3DR8 solid } \\
\text { element }\end{array}$ & 14400 & 18018 \\
\hline Reinforcement & $\begin{array}{c}\text { B31 beam } \\
\text { element }\end{array}$ & 516 & 531 \\
\hline
\end{tabular}

In the beams of the $\mathrm{BZ} / \mathrm{S} 2 / \mathrm{A}$ series there is no adhesion between the web and the flange. The parts are independent, but normal and tangential interactions in the contact surface is regarded. The "Hard" contact as the normal behaviour is assumed while the tangential behaviour included friction. The interface model of the series $\mathrm{BZ} / \mathrm{P}$ and $\mathrm{BZ} / \mathrm{P}+\mathrm{S}$ is similar but additionally the cohesive behaviour is adopted. Because of negligibly small thickness of the interface, the surface based on cohesive method is used. Its behaviour is defined using the 'traction-separation' law. The available model in the ABAQUS assumes initially linear elastic behaviour followed by the initiation and evolution of damage (Fig. 3). The elastic behaviour is defined as an elastic constitutive matrix that relates the normal and shear stresses ('traction') to the normal and shear displacement ('separations') across the interface [7, 8].

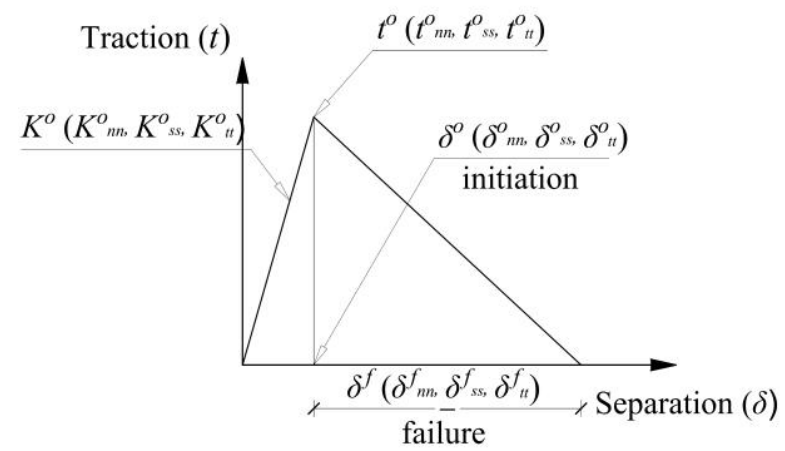

Fig. 3. Typical traction - separation response.

Damage modelling allows to simulate the degradation and eventual failure of the bond between two cohesive surfaces. The initial response is assumed to be linear. The failure mechanism description consists of two ingredients: the damage initiation criterion (described by traction $t^{\circ}$ and corresponding displacement in the interface $\delta^{o}$ ) and the damage evolution law (describing the displacement increase up to $\delta^{f}$, leading to the complete separation at failure of the surfaces connected). The nominal traction stress vector $t^{\circ}$ consists of the following components:

- normal traction $t^{o}{ }_{n n}$ and one shear traction $t^{o}$ in twodimensional problems

- normal traction $t^{o}{ }_{n n}$ and two shear tractions $t^{o}{ }_{s s}, t^{o}{ }_{t t}$ in three-dimensional problems.

The corresponding separations are denoted by $\delta_{n n}^{o}, \delta^{o}{ }_{s s}$, and $\delta^{o}{ }_{t t}$. The elastic behaviour can then be written as (1) $[7,8]$ :

$$
t^{o}=\left\{\begin{array}{c}
t_{n n}^{o} \\
t_{s s}^{o} \\
t_{t t}^{o}
\end{array}\right\}=\left[\begin{array}{ccc}
K_{n n}^{o} & K_{n s}^{o} & K_{n t}^{o} \\
K_{n s}^{o} & K_{s s}^{o} & K_{s t}^{o} \\
K_{n t}^{o} & K_{s t}^{o} & K_{t t}^{o}
\end{array}\right]\left\{\begin{array}{c}
\delta_{n n}^{o} \\
\delta_{s s}^{o} \\
\delta_{t t}^{o}
\end{array}\right\}=K^{o} \delta^{o}
$$

where $t^{o}, t_{n n}^{o}, t_{s s}^{o}, t_{t t}^{o}$, are tractions in the cohesive, $K^{o}$, $K_{n n}^{o}, K_{s s}^{o}, K_{t t}^{o}$, are the cohesive surface stiffnesses, $\delta^{o}$, $\delta_{n n}^{o}, \delta^{o}{ }_{s s}, \delta^{o}{ }_{t t}$, are the separation displacements.

\section{Results}

The analysis of the results presented below is focused on determining the proper values of parameters of the cohesive surface and their influence on the deformation and strains of the numerical model. The criterion of parameter values adjustment is high conformity to the experimental investigation results.

\subsection{Deflection}

Deflection of the beam tested is measured using linear variable differential transformer gauges located in the middle of the beam span. To compare the deflection of FEM model, the vertical axis displacement is taken.

Figure 4 presents "load-deflection" $(F-d)$ curves for the three beams of BZ/S2/A series, and three options of numerical curves, which are obtained using various interface friction parameter $\mu(\mu=0.1,0.4,0.7)$. In these beams the adhesion is completely broken by use of the PVE membrane. It can be noticed that the friction coefficient $\mu=0.4$ responds to this kind of surface. Highly similar response is achieved in comparison with experimental test for this value.

Assumption of $\mu=0.7$ leads to overestimation of the interface strength of $\mathrm{BZ} / \mathrm{S} 2 / \mathrm{A}$ beams and such value is adopted for the BZ/P series (non-reinforced interface with adhesion) and $\mathrm{BZ} / \mathrm{P}+\mathrm{S}$ series (reinforced interface with adhesion) because of existing surface roughness.

Figure 5 illustrates $F$ - $d$ curves obtained in numerical analysis of BZ/P series. Parameters of cohesive surface are: separations at failure $-\delta_{n n}^{f}, \delta_{s s}^{f}, \delta_{\mathrm{tt}}^{f}=0.1 \mathrm{~mm}$, traction stress $-t^{o}{ }_{n n}, t^{o} s, t^{o}{ }_{t t}=$ from $2.0 \mathrm{MPa}$ to $f_{c t m}$ in module of $0.2 \mathrm{MPa}\left(f_{c t m}\right.$ is the tensile strength), surface stiffness $K^{o}$ $\left(K_{n n}^{o}, K_{s s}^{o}, K_{t t}^{o}\right)$ set as default.

The damage initial stress $t^{\circ}$ has the significant impact on response of the composite beam in the FEM test. It can be observed that the first step of loading is alike in all cases. Then, depending on the initial stress value, the 
sharp drop in the force with a large increase in deflection occurs. This phenomena can be identified with the interface delamination and its ultimate load strength. The values of damage initiation stress responded to the laboratory tests oscillate between 2.2 and $2.6 \mathrm{MPa}$.

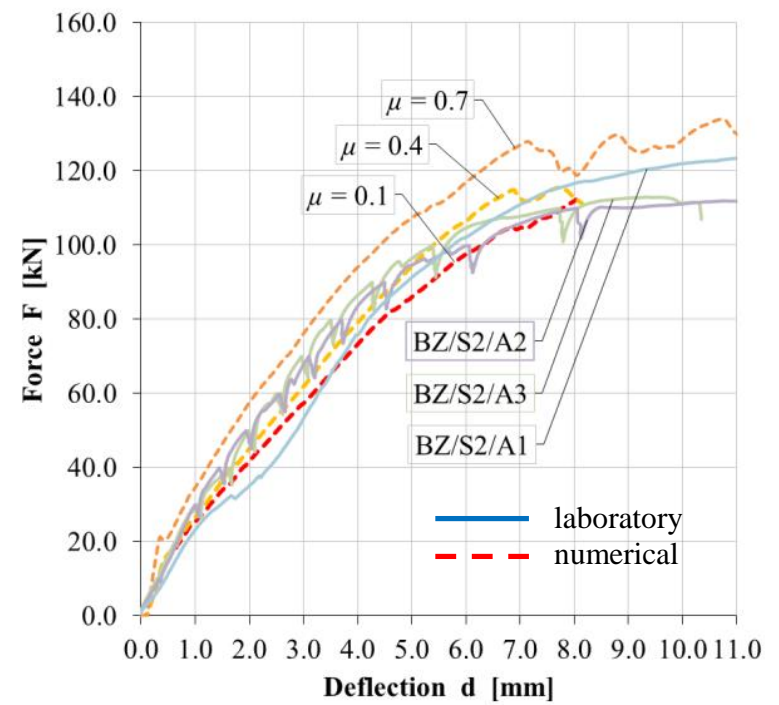

Fig. 4. Response of the FEM specimen of the BZ/S2/A series depending on the friction coefficient $\mu$.

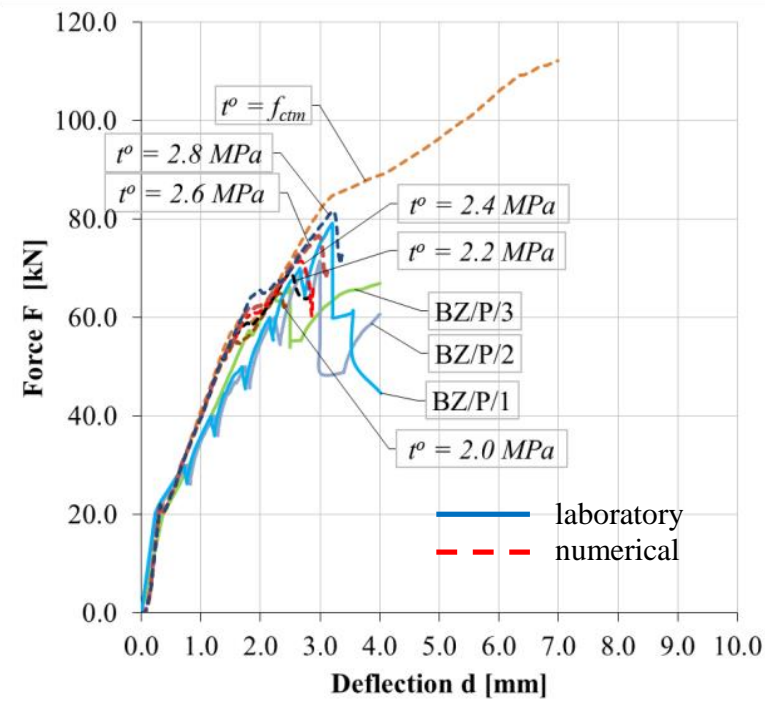

Fig. 5. Response of the FEM specimen of the BZ/P series depending on the traction stress $t^{\circ}$.

Figure 6 illustrates $F$ - $d$ curves obtained in numerical analysis of BZ/P series, depending on the separation displacement at failure $\delta^{f}$. The constant parameters of cohesive surface are: traction stress - $t_{n n}^{o}, t_{s s}^{o}, t_{t t}^{o}=2.4$ $\mathrm{MPa}$ and surface stiffness $K^{o}\left(K_{n n}^{o}, K_{s s}^{o}, K_{t t}^{o}\right)$ set as default.

Similarly to the case of initial stress $t^{0}$, separation displacement at failure $\delta^{f}$ has key impact on response of the composite beam in the FEM test. The character of the $F-d$ curve obtained in the numerical simulations is similar to that presented in the Fig.5, which is dependent on the $t^{\circ}$ parameter.

Additionally the $\delta^{f}$ has an impact not only on the critical point characterised by the interface delamination, but also the change in the $\delta^{f}$ parameter which leads to sharp peak or flattened curve. The values of separation displacement responded to the laboratory tests oscillate between 0.05 and $0.1 \mathrm{~mm}$.

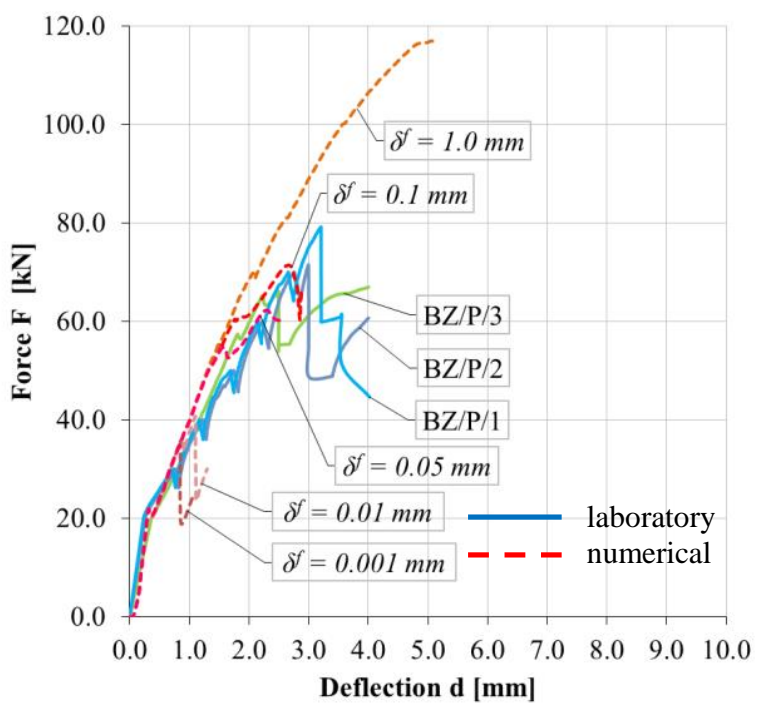

Fig. 6. Response of the FEM specimen of the BZ/P series depending on the separation displacement $\delta$.

Figure 7 illustrates $F$ - $d$ curves obtained in numerical analysis of series BZ/P depending on the surface stiffness $K^{o}$ in range 10000 to $1000000 \mathrm{~N} / \mathrm{mm}^{3}$. Other cohesive surface parameters set as: traction stress - $t_{n n}^{o}$, $t^{o}{ }_{s s}, t_{t t}^{o}=2.4 \mathrm{MPa}$ and separations $-\delta_{n n}^{f_{n}}, \delta_{s s}^{f}, \delta_{\mathrm{tt}}^{f}=0.1 \mathrm{~mm}$.

It can be observed that the $K$ parameter has insignificant influence on response of the FEM specimen. However penalty stiffness should be at least $10^{5} \mathrm{~N} / \mathrm{mm}^{3}$ in order to avoid a cohesive zone flexibility on a global compliance of the composite element. The ABAQUS user's manual recommends to set it as default, and the numerical analyses confirms this approach.

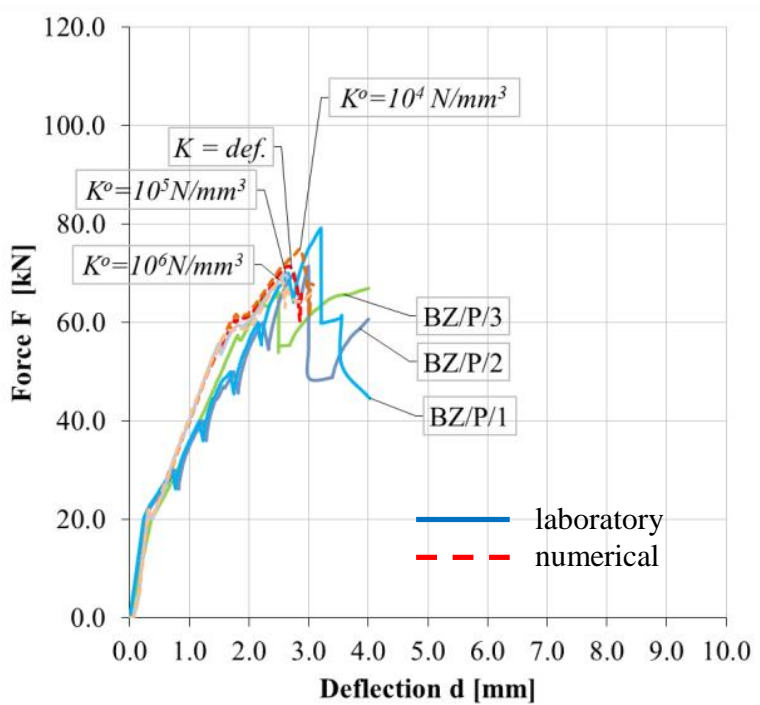

Fig. 7. Response of the FEM specimen of the BZ/P series depending on the cohesive surface stiffness $K^{o}$. 
Influence of the cohesive surface parameters $t^{\circ}, \delta^{f}$ and $K^{o}$ on the static performance of the numerical composite specimen of $\mathrm{BZ} / \mathrm{P}+\mathrm{S}$ series (with reinforced interface) is also analysed. Character of the response determined by the surface parameters is similar to the specimen of BZ/P series.

Figure 8 illustrates $F$ - $d$ curves obtained in numerical analysis of series $\mathrm{BZ} / \mathrm{P}+\mathrm{S}$ depending on the traction stress $t^{\circ}$ in range from $2.6 \mathrm{MPa}$ to $f_{c t m}=3.2 \mathrm{MPa}$. Constant parameters set as: separations $\delta_{n n}^{f}, \delta_{s s}^{f}, \delta_{\mathrm{tt}}^{f}=$ $0.1 \mathrm{~mm}$, surface stiffness $K^{o}\left(K_{n n}^{o}, K_{s s}^{o}, K_{t t}^{o}\right)=$ default.

The value of damage initiation stress responded to the laboratory test suits close to $2.9 \mathrm{MPa}$ what proved to be $0.9 f_{c t m}$. What is more, on the both $F$ - $d$ curves (numerical and experimental) it can be observed the point, which is identified as the local interface crack. At the value of about $70 \mathrm{kN}$ there is a flattening of the curve noticeable, at the same value the delamination over entire interface in series BZ/P took place. The value of traction stress $t^{o}$ has influence not only on the ultimate load but also on the interface cracking.

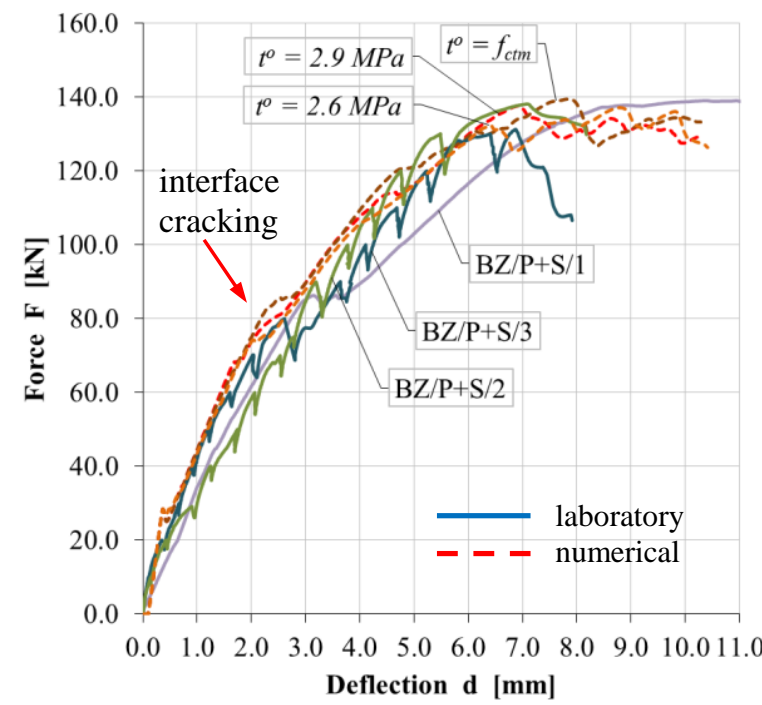

Fig. 8. Response of the FEM specimen of the $\mathrm{BZ} / \mathrm{P}+\mathrm{S}$ series depending on the traction stress $t^{\circ}$.

Figure 9 illustrates $F-d$ curves obtained in numerical analysis of $\mathrm{BZ} / \mathrm{P}+\mathrm{S}$ series depending on the separation displacement at failure $\delta^{f}$. The constant parameters of cohesive surface are: traction stress - $t_{n n}^{o}, t^{o}{ }_{s s}, t^{o}{ }_{t t}=2.9$ $\mathrm{MPa}$ and surface stiffness $K^{o}\left(K_{n n}^{o}, K_{s s}^{o}, K^{o} t\right)$ set as default.

As in the case of BZ/P series, separation displacement at failure $\delta^{f}$ has key impact on response of the composite beam in the FEM test. The character of the $F$ - $d$ curve obtained in the numerical simulations is similar to the $t^{o}$ curve. Separation displacement $\delta^{f}$ has significant impact on the ultimate and interface crack load also.

Impact of the surface stiffness $K^{o}$ on the response of specimen, like in the BZ/P series, is negligibly small. The $F-d$ curves obtained in numerical analysis depending on the $K^{o}$ parameter is shown in Figure 10.

\subsection{Strain on the reinforcement}

Strain of the reinforcement bars was measured by the electric strain gauges arranged on the stirrups and main bar as shown in Figure 1. The stirrup strain gauges were located in a line at the interface height from the outside of the perimeter; the main bar strain gauge was located in the centre of the beam span, on its bottom surface.

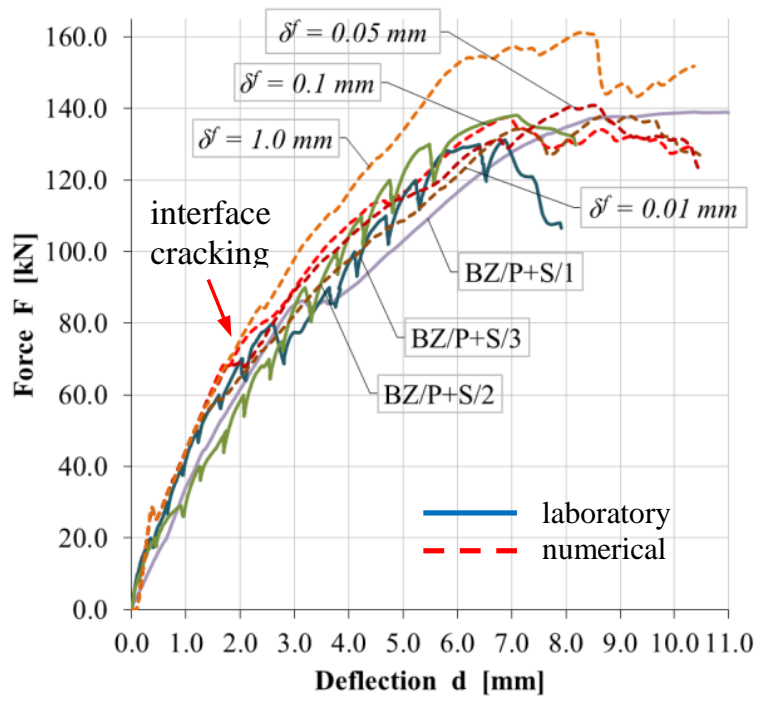

Fig. 9. Response of the FEM specimen of the BZ/P+S series depending on the separation displacement $\delta$.

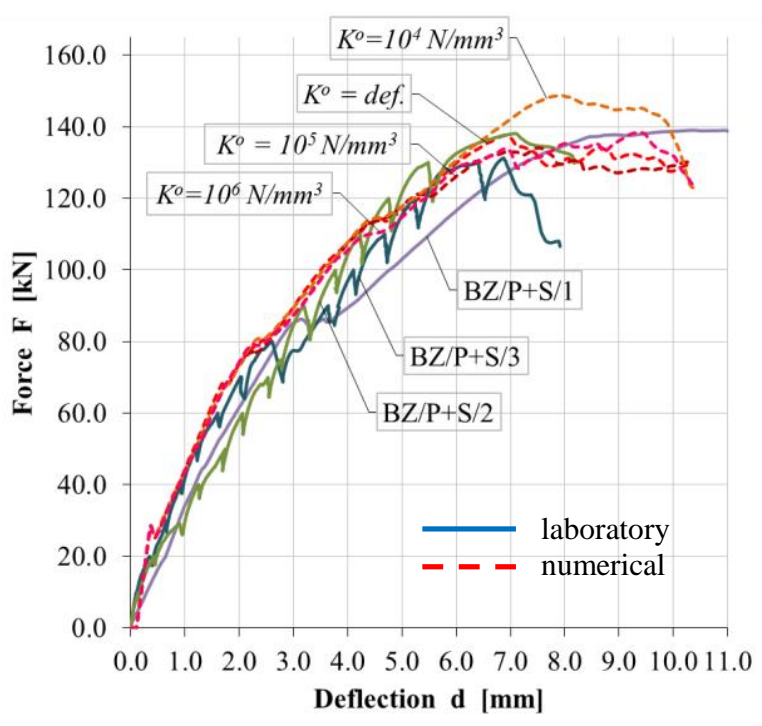

Fig. 10. Response of the FEM specimen of the BZ/P+S series depending on the cohesive surface stiffness $K^{o}$.

The increase in the strain of the main reinforcement along with the increase of the load in representative beams of all series varied is shown in Figure 11.

The chart shows that, at the beginning stages of test, the angle of "load-strain" curves obtained from numerical analyse is slightly higher than the investigated one. Then, to the end of the test, both curves are parallel and linear; the yield strength is not reach. The reason of the curves difference might be non-discrete forming of cracks in the numerical model which finally has no 
influence on a static performance of the composite element.

Attention should be paid to the performance of the transverse reinforcement in the support zone. In the BZ/S2/A series (reinforced interface and eliminated adhesion, Fig.12) an increase in strain is noticeable from the beginning of load application. In this series the activation of the stirrups Ts4 in the zone of constant moment is characteristic. In stirrups Ts4 in the other beams only little strain values in this zone arises.

Fig. 11. Response of the FEM specimen of the BZ/S2/A series

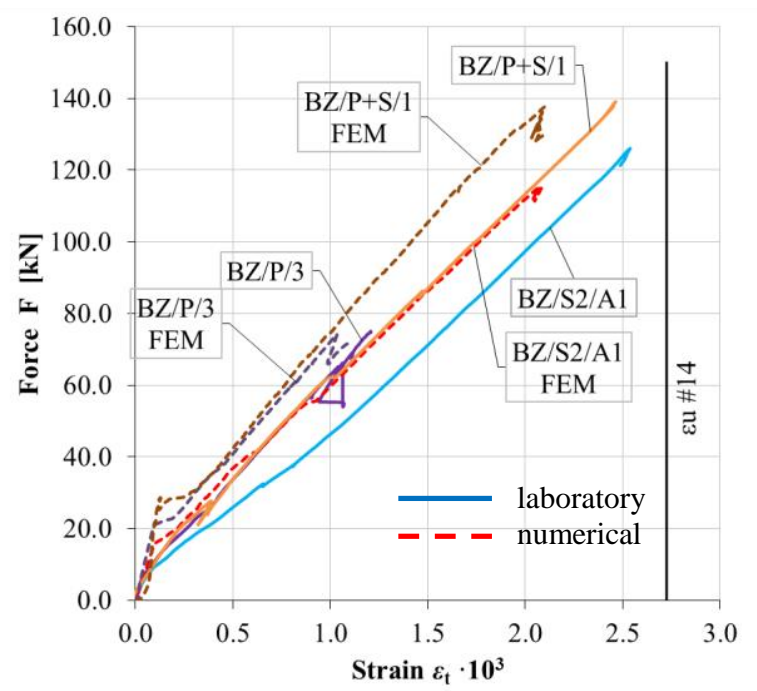

in the Ts3 strain gauge place; $\varepsilon_{\mathrm{u}} \# 14$ - strain value corresponding to the yield strength of the main reinforcement.

Fig. 12. Response of the FEM specimen of the BZ/S2/A1 series in the Ts3 strain gauge place; $\varepsilon_{u} \# 14$ - strain value

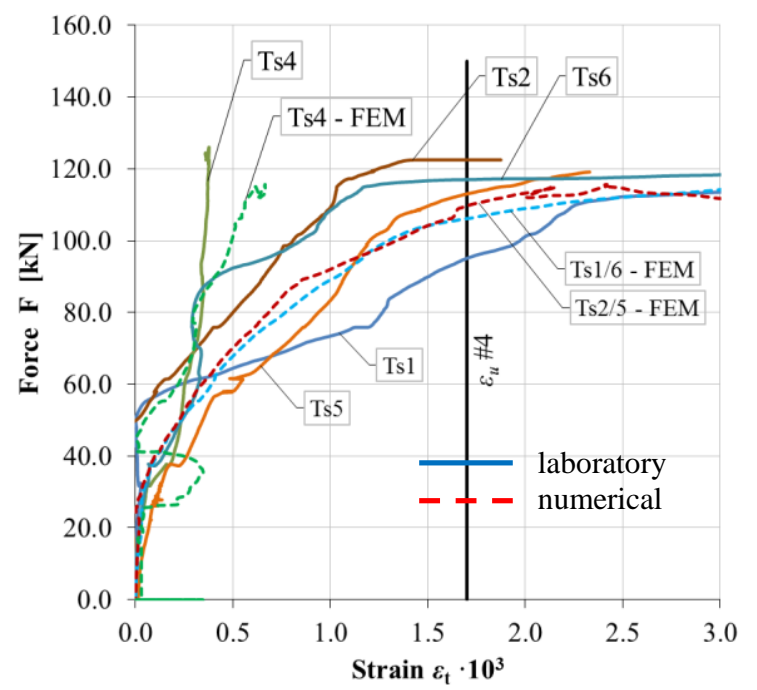

corresponding to the yield strength of the main reinforcement.

On the other hand, in the charts of the support stirrups strain (Ts2, Ts5), in the beams of the BZ\P+S series (with reinforcement and adhesion, Fig.13), a leap is visible (with a force of about: $45 \mathrm{kN}$ - investigation, $70 \mathrm{kN}$ - FEM), which can be identified with the interface crack. In further stages of the load applying, the strain in the stirrups of beams increase until the yield strength is reached. It is observed that the subsequent stirrups covered by a diagonal crack are engaged, the strain of which increases until the beam is destroyed when a higher load can no longer be applied.

Performance of the stirrups in BZ/P series (without the joining reinforcement) was a rather different. The stirrups that were formed in the mould covered only the web, and the strain gauges were located halfway up the beam height. There was a slight increase in strain after the diagonal crack appears in the web, and strain gauges broke off after the delamination of the interface.

Static performance of the reinforcement in the tests and numerical model is very similar. The same phenomena in particular series can be observed, which confirms the quality of the FEM model, with only a few disparity in value of the load caused the particular ones. In the case of transverse reinforcement, quantity compare of the numerical model and beams tested is not truly reliable, because of difficulties with the symmetry of loading and impurity of the specimen. However the modes of failure and the deflection responses of FE model are convergent with investigations so the results of numerical analyses can be used in the parametric studies successfully.

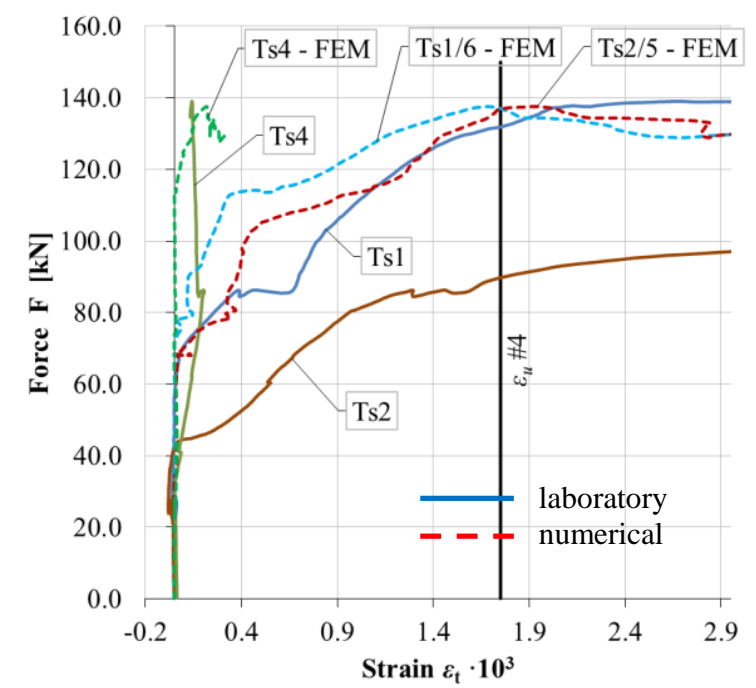

Fig. 13. Response of the FEM specimen of the BZ/S2/A1 series in the Ts3 strain gauge place; $\varepsilon_{u} \# 14$ - strain value corresponding to the yield strength of the main reinforcement.

\section{Conclusions}

On the basis of the test results and the numerical analysis, the following conclusions may by drown:

- The method of the interface formulation (cohesive surface parameters) has a major effect on the static performance of the composite beams,

- Defining the interface of concrete composite beam as a surface with normal, tangential and cohesive interactions leads to high conformity of the numerical model to the experimental investigation,

- The numerical analysis with use of the Concrete Damage Plasticity Model of ABAQUS leads to reasonable results in terms of deformation and crack pattern even without the discrete crack propagation, 
- $\quad$ Key impact on response of the composite beam in FEM test has the separation displacement at failure $\delta$. Defining proper values of the parameters $\delta_{n n}^{f}, \delta_{s s}^{f}, \delta_{t t}^{f}$ allows to achieve very good agreement of the $F-d$ curve not only regarding the ultimate load but also in its shape (sharp or flattened) identifying interface crack or delamination phenomena,

- The values of separation displacement at failure $\delta^{f}$ (for numerical analyses) should be taken between 0.05 and $0.1 \mathrm{~mm}$, and it is rather constant parameter for concrete,

- The damage initiation stress $t^{\circ}$, in the "tractionseparation" law and friction parameter $\mu$ respond to the roughness of the surface, have also significant impact on response of the composite beam in the FEM test,

- Impact of the surface stiffness $K^{o}$ on the response of specimen is negligibly small. It is recommend to set it as default or at least $10^{5} \mathrm{~N} / \mathrm{mm}^{3}$,

- The next step of the author numerical analyses will be focused on influence of the interface reinforcement ratio on the performance of the composite beams.

The research conducted indicates that numerical analysis should be consider as an invaluable tool, providing supplement and extension of experimental tests, regarding particular the visualisation of adhesive joint behaviour undergoing failure processes. Observation of such occurrences in standard experimental research proves hindered if not impossible.

This work was financially supported by the statutory budget funds of Faculty of Civil Engineering and Architecture in Lublin University of Technology.

\section{References}

1. Ł. Jabłoński, API C. P., 1, (2018)

2. A. Halicka, Ł. Jabłoński, P. I. C. E. - S. and B., 169, 67-75 (2016)

3. Ł. Jabłoński, A. Halicka, I.\&B., 7, 362:365 (2015)

4. E. Cavaco, I. Pacheco, J. Camara, E. S., 156, 210223 (2018)

5. E. Cavaco, J. Camara, E. S., 132, 278-287 (2017)

6. P. Kmiecik, M. Kamiński, A. C. M. E., 11, 623-636 (2011)

7. Abaqus User's manual Version 6.12 Dassault Systemes Simulia Corp. (2012)

8. K. Dadej, B. Surowska, C. T. P., 16:3, 180-188 (2016)

9. A. Demir, N. Caglar, H. Ozturk, Y. Sumer, E. S., 120, 158-165 (2016)

10. K. Cichocki, J. Domski, J. Katzer, M. Ruchwa, B. M. C., 11, 309-318 (2015)

11. M. Oliver, JRC S. and T. R., 56256, (2010)

12. T. Yu, J.G. Teng, Y.L. Wong, S.L. Dong, E. S., 32, 680-691 (2010) 